\title{
MÁS ALLÁ DE LA CARIDAD Y LA FILANTROPÍA: EL SURGIMIENTO DEL TRABAJO SOCIAL EN ANTIOQUIA*
}

\author{
BEYOND CHARITY AND PHILANTHROPY: THE EMERGENCE OF SOCIAL \\ WORK IN ANTIOQUIA
}

\author{
Diana Ramírez-Duarte** \\ LeIDy Karina LÓPez-PalaCiO*** \\ DANIELA URIBE-ESPINOSA****
}

\section{Resumen}

Objetivo. Presentar un análisis histórico-crítico sobre el surgimiento del Trabajo Social en Antioquia a partir de la perspectiva de totalidad. Metodología. Revisión documental de artículos de prensa en El Colombiano y documentos sobre la historia del Trabajo Social de la época, como parte de una investigación más amplia sobre la historia de la profesión en Colombia, realizada por el Colectivo de Trabajo Social Crítico Colombia. Resultados. El surgimiento del Trabajo Social en Antioquia respondió a los intereses de la clase burguesa y la iglesia católica para paliar las expresiones de la cuestión social, favoreciendo la reproducción de la fuerza del trabajo, desde la mediación de los servicios sociales regulados por las políticas estatales. Conclusión. Superar la enseñanza sobre la historia del Trabajo Social en cuanto evolución de la caridad y la filantropía, para ubicarlo dentro de la división social del trabajo y así descubrir las contradicciones propias en su devenir.

Palabras clave: trabajo social, capitalismo, Estado, iglesia, clases sociales.

\begin{abstract}
Objective. To present a historical-critical analysis on the emergence of Social Work in Antioquia from the perspective of totality. Methodology. Documentary review of press articles in El Colombiano newspaper and documents on the history of Social Work of the time, as part of a wider investigation on the history of the profession in Colombia, carried out by the "Colectivo de Trabajo Social Crítico Colombia”. Results. The emergence of Social Work in Antioquia responded to the interests of the bourgeois class and the catholic church to alleviate the expressions of the social issue favoring the reproduction of the labor force, from the mediation of social services regulated by state policies. Conclusion. To overcome the teaching on the history of Social Work as evolution of charity and philanthropy to place it within the social division of labor and discover the contradictions inherent in its evolution.
\end{abstract}

Key words: social work, capitalism, State, church, social classes

\footnotetext{
* Esta investigación fue financiada por la Corporación Universitaria Minuto de Dios - Uniminuto, y se deriva del proyecto titulado "Historia del Trabajo Social en Colombia, zona Antioquia y Eje Cafetero, análisis histórico-crítico".

** Corporación Universitaria Minuto de Dios. Bello, Colombia. E-mail: dramirezdu2@uniminuto.edu.co.

(1) orcid.org/0000-0001-8588-245 Google Scholar

*** Corporación Universitaria Minuto de Dios. Bello, Colombia. E-mail: llopezpalac@uniminuto.edu.co.

(1) orcid.org/0000-0002-0345-3115 Google Scholar

**** Corporación Universitaria Minuto de Dios. Bello, Colombia. E-mail: duribeespin@uniminuto.edu.co.

(1) orcid.org/0000-0002-9844-793 Google Scholar
}

rev. eleuthera. 20, enero-junio 2019, 157-178 
Más allá de la caridad y la filantropía: el surgimiento del Trabajo Social en Antioquia

\section{Introducción}

ElTrabajo Social en Colombia "surge como respuesta a un conjunto de condiciones económicas, sociales, culturales y políticas de orden nacional e internacional” (Martínez, 1981, p. 9). Estas respuestas aparecen en el marco del fortalecimiento de las luchas de la clase trabajadora, que comienzan a exigir mejores condiciones de vida, pero también la transformación radical del modo de producción imperante.

Esta tesis, defendida desde la perspectiva histórico-crítica, se contrasta con la ya tradicional y establecida afirmación de que el trabajo social (TS) aparece en la sociedad como la profesionalización de las formas de caridad y filantropía que se dieron en el siglo XIX en Europa y Estados Unidos.

En contraste, se busca evidenciar que es el Estado quien demanda la creación de la profesión, no como forma de ayuda, sino como "fuerza de trabajo para amortiguar las profundas condiciones de dominación y explotación en las que se encuentra la clase que vive del trabajo" (Castañeda y Ramírez, 2011, p. 14).

En ese sentido, el análisis presentado sobre el surgimiento y consolidación de la profesión en el departamento de Antioquia busca hilar, desde la perspectiva de totalidad, el desarrollo interno (sistema curricular, perspectivas teóricas, procesos de intervención) de la Escuela de Servicio Social (ESS) en la Universidad Pontificia Bolivariana (UPB) creada en 1945, con las contradicciones y dinámicas que exigían la fundación de la escuela, pasando por la influencia del sector industrial y la iglesia católica, en abierta oposición a las luchas de los y las trabajadoras.

Los resultados aquí expuestos hacen parte de la investigación "Historia del Trabajo Social en Colombia, zona Antioquia y Eje Cafetero: análisis histórico-crítico”, que tiene como objetivo: analizar la historia del TS en Colombia entre 1920 y 1979 (periodo clásico y de Reconceptualización), haciendo énfasis en las regiones de Antioquia y el Eje Cafetero a partir de los fundamentos histórico-críticos con el fin de aportar al fortalecimiento teórico de la profesión.

De este modo, los análisis desarrollados fueron retomados como parte fundamental, considerando la importancia de generar conocimiento acerca del surgimiento del TS en Antioquia, teniendo relevancia en el conjunto de la profesión en el país. 


\section{Referente teórico y conceptual}

La comprensión de los orígenes del TS en Antioquia exige el entendimiento de categorías de análisis macro para generar mediaciones entre la conformación y desarrollo de las escuelas de TS, con los acontecimientos y sucesos que influyen en las mismas y determinan su devenir.

Desde la perspectiva de la totalidad concreta, se entiende así la formación socioeconómica capitalista, como los procesos económicos, sociales y políticos derivados de la instalación del modo de producción capitalista. En Colombia específicamente, según Fajardo (2013) esta presenta siete características: acumulación por desposesión; crisis mundial (ambiental y climática); crisis del régimen político; transnacionalización y desnacionalización (dependencia al imperialismo); imposición de valores genuinamente capitalistas y dimensiones transnacionales de la cuestión armada.

El estudio dialéctico de la realidad concreta colombiana permite vislumbrar las contradicciones propias de la formación socio-económica capitalista con relación a las clases sociales, teniendo como base la afirmación de Marx y Engels (1999): “Toda la historia de la sociedad humana, hasta la actualidad, es una historia de luchas de clases" (párr. 1); se comprende que esta frase contiene una riqueza teórica enorme, pues expresa la relación, desde la perspectiva de totalidad, entre superestructura y estructura.

Al mencionar las clases, Marx y Engels se refieren a la formación económica de las relaciones sociales, es decir, los procesos de producción y su influencia en las relaciones sociales. En otras palabras, las clases sociales se conforman a partir de los modos de producción que se encargan de generar diferentes grupos sociales (las clases) con relación a su posición dentro de la producción. Esto tanto en el esclavismo y en el feudalismo como en el capitalismo.

Por otro lado, la conformación de estos grupos sociales se ha realizado de forma jerarquizada, al responder a diferentes aspectos históricos, culturales y económicos. Así, en el feudalismo el poder era heredado, es decir, la familia determinaba en gran parte la posición social del individuo. Ya en el capitalismo es el mercado y la participación en la división del trabajo lo que ubica a una persona en una u otra clase social.

Pero esta jerarquización, es decir, la existencia de clases sociales, trae al mismo tiempo una confrontación entre ellas, particularmente por la búsqueda de emancipación de la clase dominada. Así, la clase dominante (generada por la estructura/esfera económica) utiliza todas las herramientas a su alcance para continuar en esta posición, que tiene relación, directa o indirectamente, con el poder (superestructura/esfera política). 
Por tanto, al analizar el objeto de estudio específico que guía esta investigación, también se hace esencial comprender la categoría de análisis de instituciones sociales, teniendo dos centrales: el Estado y la iglesia. El Estado, como producto irreconciliable de las clases sociales, es una institución de poder público, que se organiza como fuerza armada y separada del resto de la población; además, concentra la riqueza de cada nación, volviéndose en instrumento de explotación de la clase oprimida (Lenin, 2003). Para el catolicismo, por el contrario, la Iglesia es el "Estado universal y sobrenatural" (Gramsci, 2002, p. 226). Aquí entonces es necesario entender la sociedad civil como el aparato privado de hegemonía del Estado, pero además, como espacio de disputa entre clases, pues allí se encuentran instituciones como la escuela, la iglesia, los medios de comunicación... etc. (Gramsci, 2002).

En el camino del entendimiento de las realidades concretas, también aparece una categoría de análisis esencial: territorio. Este permite comprender la necesidad de tener en cuenta la producción del espacio como disputa territorial de las clases sociales antagónicas. La realidad concreta y sus particularidades, también se deben dilucidar en las condiciones específicas del territorio, comprendiendo dinámicas propias, en este caso, de Antioquia y Colombia. Así, el territorio, desde la concepción relacional, se refiere a un fragmento del espacio (producido socialmente) de un grupo social, que no es fijo ni impermeable, y es determinado por las relaciones sociohistóricas y, por tanto, por las relaciones de poder (Renaud, 2015). "La construcción de este se da a partir de una relación dialéctica compuesta por verticalidades y horizontalidades que hacen de este territorio de consensos y de disputas" (Castañeda, 2016, p. 51).

Por último, la categoría de análisis de educación es utilizada en esta investigación desde los procesos formales en relación con la creación y consolidación de las escuelas de TS, los currículos, las perspectivas teóricas, la práctica profesional y la conformación de las universidades en el país y la ciudad. Sin embargo, en una concepción amplia, esta no puede solo referirse a las dinámicas propias de enseñanza-aprendizaje: "Por educación tendríamos que entender todo el conjunto de procesos en los que son incardinadas las personas, y en el contexto de los cuales - y bajo cuyo condicionamiento - tienen lugar su socialización y su individuación” (Acanda, 2004, p. 33). De igual forma, se comprende la educación como proceso determinado y determinante por los sujetos, es decir, como espacio de posibilidad para la transformación social.

\section{Metodología}

La utilización de las categorías de análisis como mediaciones teóricas para analizar la realidad parte de comprender la totalidad concreta "1) como articulación entre conceptos de diversos niveles de abstracción, 2) como articulación entre lo lógico y lo histórico, 3) como articulación de procesos de diversas temporalidades, 4) como articulación entre subjetividad y objetividad” (De la Garza, 1993, p. 137). 
De esta manera, a partir de cada objetivo específico se derivan las categorías macro desarrolladas en el apartado anterior (formación socioeconómica capitalista, clases sociales, instituciones, territorio y educación), de las cuales se desglosan subcategorías y elementos empíricos observables, definidos como aquellas unidades de análisis mínimas para entender el objeto de estudio.

En el proceso metodológico, por lo tanto, no solo se tienen en cuenta los hechos estructuralmente, sino también los procesos de los sujetos colectivos que, en medio de las contradicciones sociales, políticas y económicas, generaron tensiones que influyeron en el curso de la historia. Además, la comprensión de los espacios geográficos y las temporalidades, bajo las diferentes categorías, dan cuenta de la necesidad de analizar este objeto de estudio más allá de los procesos formales-legales y los desarrollos curriculares propios de la ESS.

Las fuentes de información utilizadas para el análisis del surgimiento del TS en Antioquia fueron secundarias (investigaciones, artículos, ponencias y libros) y de diversa índole, teniendo en cuenta que sobre la historia de la profesión en este departamento, se encuentra poca bibliografía reciente, lo que llevó a la necesidad de ir a fuentes documentales primarias (documentos jurídicos y normativos, y noticias de prensa) sobre el contexto social, político, cultural y económico de Colombia y Antioquia.

A partir de la técnica de revisión documental, en la que se destacan las noticias del periódico El Colombiano de Medellín, en años comprendidos entre 1920 y 1952, claves para la comprensión de la creación y consolidación de la ESS en la ciudad; se realizó el análisis de la información bajo la comprensión del movimiento de esta realidad concreta, comprendida como síntesis de múltiples determinaciones, que tiene en su centro el análisis histórico-dialéctico (contradicciones entre aspectos de la realidad social) y material (reproducir el objeto real en la mente por medio de la abstracción).

Este análisis se llevó a cabo desde discusiones con el Semillero de Trabajo Social Crítico de la Corporación Universitaria Minuto de Dios-Seccional Bello, las autoras y el Colectivo de Trabajo Social Crítico Medellín ${ }^{1}$.

\section{Resultados}

Entre 1920 y 1940 Antioquia fue protagonista en el desarrollo industrial y urbanístico del país a través de la explotación minera, la producción de café y la industria textilera, principalmente. En el departamento se comenzaron a instalar políticas de bienestar social en áreas rurales y urbanas, resultado tanto de los procesos de urbanización y pauperización propios de la

\footnotetext{
${ }^{1}$ Perteneciente al Colectivo de Trabajo Social Crítico Colombia.
} 
formación socioeconómica capitalista, como de las contradicciones agudizadas por la naciente clase obrera y sus luchas.

La ESS se funda en 1945 con un carácter técnico-asistencial y lineamientos religiosos, alienada con los intereses de las clases dominantes para el ejercicio del control social, asentando sus acciones en modelos internacionales, convirtiéndose así en una profesión indispensable para la intervención social, que concentraba inicialmente sus labores en las industrias. Entre 1943 y 1946 se fortaleció el proceso de industrialización en Antioquia y, a su vez, el flujo de importación y exportación de mercancías. En medio de las disputas liberales y conservadoras se dio la alianza entre el Estado, la iglesia, y las entidades privadas, para asistir a las exigencias del contexto por medio de las políticas sociales estatales. También confluyen las expresiones del proletariado por medio de manifestaciones y aspiraciones políticas contrarias.

Entre 1950 y 1960 se comenzó a dar reconocimiento político a la clase obrera, debido a las constantes luchas por la reivindicación de sus derechos, momento en el cual algunos grupos insurgentes ya existían y al mismo tiempo tomaban fuerza. Asimismo, las relaciones internacionales continuaban y su injerencia enfocada en la economía y la política del país. La intromisión del TS en los campos de acción, permitió visibilizarse en la sociedad y también constituir un desarrollo importante para la profesión, junto con el fortalecimiento de los procesos gremiales.

\title{
Discusión de resultados
}

\begin{abstract}
El capitalismo (...) nace en Colombia en los últimos años del siglo XIX y se consolida en el siglo XX. Este se desarrolló sobre las bases de un capitalismo dependiente, que, en sus comienzos, se debió a la gran afluencia de las importaciones (especialmente manufacturas) y los respectivos impuestos aduaneros, lo que daba como resultado el poco desarrollo de actividades económicas internas, con excepción del café. (Ramírez, 2014, p. 22)
\end{abstract}

Antioquia ha sido considerada una región avanzada industrialmente gracias a actividades económicas como la minería, el café, el comercio, la ganadería y la industria textilera, siendo parte de "la acumulación de capital por parte de una variada gama de sectores que configurarían luego a la naciente burguesía industrial” (Vega, 2002, párr. 1). La exportación de maquinaria en el país se hizo inicialmente en este departamento en 1904, junto con la importación de materias primas (Vega, 2002). La década del 20 se caracterizó por la creación de sociedades anónimas y el fortalecimiento del mercado financiero (Melo, 1988).

Paralelamente, se construyeron obras de infraestructura vial (Gómez, 2001) y se inició la segmentación espacial, separando internamente las ciudades (incluyendo Medellín) entre los barrios obreros y los barrios de las clases dominantes (Vega, 2002). 
En otra arista, se intensificaron las protestas cívicas en Colombia. En Antioquia se dieron en contra del aumento de impuestos al comercio, la violencia y la represión estatal. También se dieron diferentes luchas particulares de la clase trabajadora. Dentro de estas, es necesario recordar la "Masacre de las bananeras" en 1928 en el Urabá y la primera huelga general en Colombia, realizada en la Fábrica de Tejidos de Bello en 1904, dirigida por Betsabé Espinal y protagonizada por 400 obreras. Igualmente, se destaca el papel de María Cano, quien fue nombrada Flor del Trabajo, por su protagonismo en la lucha sindical y obrera.

Por otro lado, para contrarrestar los procesos de urbanización y pauperización, a finales de la década del 20 se crearon algunas instituciones filantrópicas por diferentes actores sociales (Melo, 1988). En Medellín, la asistencia social estatal se dirigió a promulgar políticas de vivienda, casas y juzgados de menores y hospitales estatales ${ }^{2}$ (El Colombiano, 1920).

En el aspecto educativo, en 1926 se establecieron las condiciones para ingresar a las facultades universitarias oficiales, en 1927 se fundó el Ministerio de Educación Nacional, como fusión de dos ministerios (de instrucción y de salubridad), y se fomentó la creación de escuelas domésticas para mujeres en 1928.

Martínez (1981) establece que entre 1920 y 1930 se da el surgimiento de la política social, como respuesta del Estado a peticiones y luchas de los trabajadores y la reproducción de la fuerza de trabajo. Esta también genera división social del trabajo, aplicando límites de acción al bienestar social, priorizando vivienda, salud y educación; se centra en la ciudad y su aplicación se da, primariamente, por el sector privado y la iglesia.

Luego de la crisis de la bolsa de Nueva York en 1929, en Colombia se vio una baja en las importaciones y en el precio del café, el aumento del desempleo, al igual que la quiebra de varias empresas y la disminución de la construcción de obras públicas ${ }^{3}$.

De esta manera, durante los gobiernos de la República Liberal (1930-1946) en la década del 30, el Estado comenzó a intervenir lo monetario y lo financiero, reforzando el proceso de sustitución de importaciones y el equilibrio en la balanza de exportaciones (Melo, 1988). También se creó la "Ley de Reforma Agraria" y la Reforma Constitucional, que abarcaron la intervención estatal en la economía y la asistencia pública (garantizar los mínimos de sobrevivencia y el trabajo como obligación social) (Botero, 2006). Esto estuvo acompañado del fortalecimiento de la "enseñanza industrial" y de la fundación de internados de educación campesina gratuita.

\footnotetext{
${ }^{2}$ Esto en el marco de la Ley 15 de 1923, que regulaba las Casas de Menores y Escuelas de Trabajo. El programa Gota de Leche también fue fuertemente promovido por el gobierno nacional bajo la ley 43 de 1928.

${ }^{3}$ En medio de la crisis, entre 1931 y 1933 se desarrolla la guerra colombo-peruana, por la invasión del Perú al Sur de Colombia, y a nivel mundial se asiste al ascenso de Hitler al poder en Alemania en 1933 y en 1936 comienza la Guerra Civil Española, reforzando proyectos políticos dictatoriales en el continente europeo.
} 
Más allá de la caridad y la filantropía: el surgimiento del Trabajo Social en Antioquia

Todas estas reformas económicas y el intervencionismo estatal que se dieron con el comienzo de la "República liberal" (1930-1946), llevan al conservadurismo a ejercer acciones de abstención y a promulgar variadas manifestaciones en contra del gobierno liberal (Melo, 1988).

En contraste, a partir de 1931, el liberalismo comienza a promover movilizaciones populares de sectores de izquierda, revolucionarias, de estudiantes y obreros, lo que lleva al aumento de las movilizaciones en la ciudad de Medellín (Melo, 1988). De esta manera, las organizaciones comunistas y socialistas no logran fortalecerse en la ciudad y dan paso a grupos, sindicatos y procesos colectivos de corriente liberal, tal como la Confederación Nacional de Trabajadores (CTC), creada en 1935.

Sin embargo, el Partido Comunista Colombiano (PCC), fundado en 1930, logra la elección de dos concejales en el Concejo de Medellín en este mismo año, asunto que no logra mantenerse en el tiempo. Por esto, y con el objetivo de asumir la política del Frente Popular tomada por la III Internacional Comunista, a finales de este año, el PCC buscó alianzas con el gobierno de López Pumarejo y con una "imaginaria burguesía progresista” (Melo, 1988, p. 157). En Medellín esto no logra concretarse, perdiendo gran fuerza política.

La consolidación de este proyecto económico y político liberal también estuvo marcada por manifestaciones y huelgas de diferentes tipos, como las estudiantiles y de la clase trabajadora (huelgas en Ecopetrol, Rosellón y Coltejer) ${ }^{4}$.

Por su parte, la iglesia católica antioqueña reforzó sus posiciones antiliberales y anticomunistas, con el protagonismo del monseñor Miguel Ángel Builes de Santa Rosa de Osos y la celebración del Congreso Eucarístico Nacional (1935) (Melo, 1988).

La primera Escuela de Servicio Social fue creada entonces en 1936 en el Colegio Mayor de Nuestra Señora del Rosario, por María Carulla de Vergara, bajo la orientación de la Unión Católica Internacional de Servicio Social (UCISS) -creada en 1925 en Roma- (Martínez, 1981) y la Encíclica Quadragesimo anno de 1931, que llamaba a la conciliación de clases, haciendo énfasis en los cristianos que tenían un proceso de cualificación científica y profesional (Quintero, 2014). También en 1936 se fundó la Universidad Pontificia Bolivariana (Jaramillo, 1996). Ya en 1940 se otorga el reconocimiento oficial a la primera ESS con la Resolución 317 (Quintero, 2014).

En la década del 40, el Estado continuó fortaleciendo la política de sustitución de importaciones y comenzó a hacer alianzas entre el sector privado y el estatal (capital mixto) (Melo, 1988).

\footnotetext{
${ }^{4}$ Una de las victorias alcanzadas fue la reducción de la jornada laboral a 8 horas diarias mediante la Ley 10 de 1934 (Jaramillo, 1996).
} 
La producción de capital empezó a ser controlada por medio del impuesto a la industria, el consumo de materias primas por parte de las industrias (El Colombiano, 1948g), el impulso de la producción agrícola y cafetera, y la adecuación del "sector agrario a las exigencias de la industria" (Martínez, 1981, p. 46).

En ese sentido, algunos productos agrícolas, especialmente el café, fueron clave del desarrollo económico del país por su movimiento de exportación a Estados Unidos, generando así una cada vez más avanzada dependencia económica. Además, se identificó que a raíz de la migración que se produjo en el área rural por causa de la violencia, los capitalistas se vieron obligados a generar una serie de políticas proteccionistas para los agricultores, con el fin de retomar las labores en el campo. Otra forma de acumulación estuvo centrada en el extractivismo, poniendo en declive las condiciones ambientales y, a su vez, las de la población.

En Antioquia, en este periodo, el café continuaba siendo el principal producto de exportación y las fuentes petrolíferas eran un centro de atracción, al igual que los metales y la ganadería con elevados capitales, desde donde también se financiaron las plantas de energía.

Por otro lado, el proceso de industrialización produjo un fenómeno de movilidad social de considerable magnitud, pues las personas que habitaban los campos vieron posibilidades de sustento en las fábricas que se estaban creando en Medellín, por tal razón, emprendieron migraciones hacia estos lugares ${ }^{5}$.

La política social en esta época tuvo un avance respondiendo a la necesidad de impulsar el desarrollo económico y a la presión de la clase obrera (en el país y en el resto del mundo), entrando “en un proceso de institucionalización” (Martínez, 1981, p. 54).

De esta manera, el sector oficial empieza a tomar fuerza frente a la iglesia católica y el sector privado, sobre todo en el campo de la vivienda y la salud. Sin embargo, los programas sociales estaban manejados por las clases dominantes, organizadas en los partidos liberal y conservador y las grandes asociaciones gremiales: Asociación Nacional de Industriales (ANDI), Federación de Cafeteros (FEDECAFE) y Federación Nacional de Colombia (FENALCO) (Martínez, 1981).

Por su parte, el gobierno departamental intervino fuertemente con programas en el área de la educación rural, la salud ${ }^{6}$, la protección a la infancia, la vivienda urbana y rural y la garantía

\footnotetext{
${ }^{5}$ Cabe resaltar que se considera el desarrollo urbanístico en Colombia y específicamente en Antioquia, como otro tipo de violencia que estuvo relacionado directamente con la llegada del capitalismo al país a comienzos del siglo XX y la llegada de los campesinos a la ciudad, todo esto en el proceso de acumulación originaria.

${ }^{6}$ Institucionalizada como servicio social con el Instituto de Seguros Sociales (ley 90 de 1946), con la cual se implementan políticas de bienestar social hacia la clase trabajadora (Martínez, 1981).
} 
Más allá de la caridad y la filantropía: el surgimiento del Trabajo Social en Antioquia

de derechos laborales (El Colombiano, 1944b, 1944g, 1944k, 1944m, 1945b, 1945d, 1946b, 1947c).

En medio de esta búsqueda por la aceleración de la industria y la intensificación de las luchas sociales de diferente índole, en 1943 Cecilia Echavarría Toro, hija de uno de los dueños de Fabricato, inició los trámites para la fundación de la ESS. Expuso su propuesta en el "Centro de Estudios”, lugar donde se reunían algunas mujeres de la clase burguesa de Medellín, con el fin de estudiar las cuestiones sociales (Sandino, 1987).

Por otro lado, a mediados de los años 40, el gobierno nacional fomentó la creación de Colegios Mayores de Cultura Femenina. Esto implicó que las mujeres tuvieran la posibilidad de capacitarse en diferentes campos para el mercado laboral, constituyendo para el TS uno de los puntos de partida en la conformación de las escuelas. "Cabe anotar que también se produjo la consolidación de posiciones sociales de clase media, debido a que el sector privado, generalmente de religiosas, se estableció para atender a los grupos elitistas" (Martínez, 1981, p. 91).

Finalmente, en 1945 se creó la ESS adscrita a la Normal Antioqueña de Señoritas con la resolución 1216, por iniciativa de la Arquidiócesis de Medellín y la Asociación Nacional de Industriales (ANDI) (Jaramillo, 1996).

El momento entre 1938 y 1945 es identificado por Martínez (1981) como

La segunda etapa del desarrollo de la profesión, que corresponde a un proceso de institucionalización a nivel nacional, esto obedece fundamentalmente al desarrollo del capitalismo monopólico al interior del país y a su vinculación mundial en una fase más avanzada: el imperialismo. (p. 146)

El periodo clásico del TS (desde 1936 hasta el inicio de la Reconceptualización), estuvo marcado por un carácter técnico-asistencial con orientación médica y jurídica (Jaramillo, 1996). Igualmente, se sitúa en el proceso de la reproducción de las relaciones sociales, principalmente como una actividad auxiliar y subsidiaria en el ejercicio del control social y en la difusión de la ideología de las clases dominantes en relación con las clases trabajadoras, con un carácter eminentemente religioso.

Los nexos que tenía la iglesia con elTS se denotaban también con la participación de esta en la junta directiva de la ESS, conformada también por representantes de la industria. Además, en 1945, la iglesia concretó su trabajo en el campo social, con la creación de la Organización Católica Social Arquidiocesana (OCSA), la cual articula las actividades formativas del secretariado de 
la pastoral social de Medellín y las acciones asistencialistas de la pastoral caritativa (Jaramillo, 1996), encargándose del trabajo con obreros, marginados y campesinos, así como de las obras sociales y de asistencia. Esta organización también hizo parte fundamental de la conformación, financiación y consolidación de la ESS de Medellín.

La ESS inicia como un programa postsecundario con tres años de duración. Las primeras asignaturas se fundamentaron en la doctrina social de la iglesia, la salud e higiene, el componente doméstico, aspectos generales en psicología, economía, derecho y sociología, más estrictamente de carácter técnico y operativo (Jaramillo, 1996). Estas, y los estatutos estimados por la ESS tenían una notoria y alta influencia de Europa, Norteamérica y las industrias, desligándose de las luchas sociales y las necesidades más sentidas de las clases populares.

Para 1946 el ejercicio de la profesión se consolida a través de la práctica de las primeras asistentes sociales en dos campos de intervención específicos, el primero y más incidente fue en las industrias, generando programas de asistencia a los obreros/as y sus familias, reforzando la labor de los clérigos industriales con programas de capacitación y educación moral. El segundo, la asistencia social en zonas marginadas de la ciudad con las residencias sociales (Jaramillo, 1996).

Este impulso y consolidación nacional e internacional de la profesión no estaba exento de la influencia de Estados Unidos "en el campo de las políticas de desarrollo y bienestar social" (Martínez, 1981, p. 203), ubicando al TS como recurso humano para la ejecución de planes, programas y proyectos de las políticas sociales.

En esta línea, se legitima y reconoce la acción estatal del TS como herramienta tecnificada y preparada para la intervención social a través de ley 25 de 1948, que decreta la creación de Escuelas de Servicio Social en ciudades de más de 50.000 habitantes, y la implementación de un plan de estudios revisado y vigilado por el Ministerio de Higiene y Educación. Además, con la Resolución 1216 del 16 de julio de 1947, el Ministerio de Educación aprueba oficialmente la ESS en Medellín, junto con los programas de práctica y los pénsum (Jaramillo, 1996).

Estos desarrollos de la ESS presentados, se dan en medio de grandes disputas por el poder económico, político y cultural entre los dos partidos políticos tradicionales y las ideologías que los alimentaban. El partido conservador retomó fuerza a través de la alianza de los miembros del partido, con el fin de acabar con las ideologías opuestas como el 'comunismo', que venían teniendo acogida importante en la ciudad (El Colombiano, 1944f, 1944j, 1944l, 1944n, 1946e, 1947a, 1947d, 1947g, 1948a).

El conservatismo tenía el respaldo de la iglesia católica, que ayudaba a divulgar la política conservadora antioqueña con intenciones denigrantes hacia el partido liberal. Sin embargo, 
Más allá de la caridad y la filantropía: el surgimiento del Trabajo Social en Antioquia

“el verdadero obstáculo para un posible triunfo gaitanista en Antioquia no radicó entonces en la supuesta dominación conservadora de la región, sino, irónicamente, en la tendencia 'conservadora' de los dirigentes 'liberales'” (Melo, 1988, p. 168).

De este modo, en 1946 el partido conservador obtiene la presidencia con Mariano Ospina Pérez, quien dio fuerza al incremento de la economía por medio del fortalecimiento de la agricultura (Brando, 2017), para lo cual se establecieron una serie de campañas para expandir los cultivos y a su vez, las políticas proteccionistas a los grandes agricultores. Esto originó importantes conflictos agrarios protagonizados por los campesinos, colonos y arrendatarios durante la década del 40 (Vega, 2002).

Por su parte, en las ciudades surgieron otro tipo de conflictos como resultado del aumento de la población, pues se comenzaron a exigir servicios públicos, construcción de obras de beneficio social, viviendas adecuadas, abastecimiento de recursos y alimentos y, además, se rechazó "la acumulación de riqueza por parte de los reductos minoritarios de las clases dominantes" (Vega, 2002, p. 13).

En paralelo, desde 1944 la ANDI, por medio de la Organización de los Industriales, con sede principal en Antioquia, impulsaría el crecimiento del desarrollo industrial sobre todo en la zona central del departamento, además, la llegada de conocimientos técnicos, maquinarias y equipos dieron paso a la consolidación de mayores industrias, con las que se inició un proceso de mecanización en la fabricación de los productos (El Colombiano, 1944c, 1944e, 1944h, 1945c, 1946a, 1946d).

Durante el decenio del 40 se proyectó sobre el país un agudo proceso inflacionario que agravó la situación económica de las masas trabajadoras y "se presenta un descenso en los salarios para trabajadores de diversa calificación” (Medina, 1984, p. 54).

Así, se daba el fortalecimiento de la clase trabajadora (clase para sí), que venía pronunciándose por medio de protestas que no solamente involucraban a trabajadores asalariados sino a un amplio grupo de personas pertenecientes a las "clases subalternas", como los artesanos, los campesinos, los indígenas y los pobladores urbanos que vivían en espacios diferentes a aquellos en los que se desenvolvía la relación capital-trabajo (Vega, 2002).

Por lo tanto, las expresiones huelguistas dan pie a la constitución de sindicatos en Medellín; a estos se suman las manifestaciones de diferentes sectores de trabajadores que esperan crear un mecanismo de presión que permita una reivindicación económica salarial justa (El Colombiano, 1944a, 1944i, 1945a, 1946c). Este momento también corresponde a la institucionalización y regulación del sindicalismo y los movimientos obreros con la Ley sexta de 1945. 
Las formas de represión y control en la década del 40 se caracterizaron por el control militar de las huelgas y manifestaciones, vistas en muchos casos como subversivas (El Colombiano, 1947b, 1950b). Por otro lado, en el caso de Antioquia, los trabajadores fueron destituidos y otras veces retenidos por la policía (El Colombiano, 1947e, 1947f).

Otras de las demandas que se evidenciaron en el contexto del departamento de Antioquia fueron realizadas por los/as estudiantes, quienes comenzaron a tener aspiraciones políticas y educativas ajenas a las tradicionales y las impuestas por el sistema por ello, se desencadenaron repetidas protestas universitarias, que fueron enfrentadas por el Estado y la Iglesia por medio de acciones violentas y con amenazas de cancelar sus matrículas (El Colombiano, 1944d, 1948d, 1949b, 1949c).

Sin embargo, la organización estudiantil no hizo parte en su totalidad de la oposición, pues en ese momento se encuadró el movimiento universitario conservador y sus directivas organizaban las masas estudiantiles derechistas (El Colombiano, 1948f).

La influencia de las escuelas europeas y norteamericanas en el sistema curricular de la ESS de Medellín debe vincularse con el fortalecimiento de las relaciones de Colombia con otros Estados u organismos internacionales en la época, que incursionaban en las decisiones y las reformas dentro del país. Por tanto, se comprende que la dominación imperialista de Estados Unidos, "no se trató solamente de una típica imposición sino de un acuerdo tácito, en el que la burguesía colombiana aceptó como una condición necesaria de la modernización capitalista la creciente injerencia de Estados Unidos en los asuntos domésticos del país” (Vega, 2002, párr. 1). En Medellín, a finales de la década del 40 se presentaron fuertes intervenciones extranjeras en el proceso de industrialización, desde las empresas privadas, diferentes gobiernos y organismos multilaterales como el Banco Mundial y la OEA.

En continuación, con los enfrentamientos políticos entre liberales y conservadores, y durante el gobierno de Mariano Ospina Pérez, aconteció el asesinato de Jorge Eliécer Gaitán, el día 9 de abril de 1948. Melo (1988) relata que:
(...) la furia popular que explotó a raíz del asesinato de Gaitán no se sintió con mucha intensidad en Antioquia; no hubo muertos en la capital antioqueña, aunque fue quemada la sede de La Defensa, hubo un atentado contra El Colombiano y se incendiaron y saquearon negocios en Medellín. (p. 169)

Este hecho marcó el inicio del enfrentamiento armado entre los dos bandos y desató una serie de disputas políticas e ideológicas con los grupos comunistas y socialistas del país, llevando incluso al rompimiento de relaciones con Rusia en ese mismo año y a la expulsión de agentes 
Más allá de la caridad y la filantropía: el surgimiento del Trabajo Social en Antioquia

soviéticos (El Colombiano, 1948b). Esto derivó también en manifestaciones de sectores comunistas en la ciudad.

Así, el gobierno decretó el Estado de sitio en todo el país y continúo las represalias por parte del Estado para mantener el control social como solución a la violencia. Por otra parte, la creación de partidos políticos y movimientos de izquierda se intensificó en este periodo y, a su vez, la oposición por cuenta del Estado, el conservatismo y la iglesia, que planearon una estructura sociopolítica contra la ideología comunista (El Colombiano, 1947b, 1948c, 1948e, 1949a, 1949d, 1949e, 1950e).

En este momento es cuando se identificó el comienzo del reconocimiento delTS en la ciudad, con la salida de las primeras cinco egresadas de la ESS y posteriormente, en 1949, con la asistencia de Amanda Gómez a los Congresos Panamericanos de Servicio Social (Jaramillo, 1996). También se fortalecieron vínculos con otras escuelas de TS en América Latina y el mundo. Igualmente, su reconocimiento se venía evidenciando públicamente en artículos de El Colombiano, que divulgaban el recuento histórico de la escuela, las inscripciones y matrículas y la apertura de nuevos cursos (El Colombiano, 1950a, 1950d, 1951a, 1951b, 1951c, 1951e).

Esto acompañado de la institucionalización de los servicios sociales, que se fortaleció con la creación del Departamento de Servicio Social Municipal en 1953. Tenía como finalidad "velar por el mejoramiento social de las personas al servicio del Municipio. (...) Así mismo, extender su acción social a todos los establecimientos de beneficencia, instituciones y centros asistenciales de carácter municipal” (Jaramillo, 1996, p. 34).

En 1954 se dio la creación del SENDAS (Sistema Nacional de Asistencia Social) por el decreto 2675. Este influenció masiva y profundamente al TS en Colombia, al Departamento de Servicio Social Municipal y al Departamento de Organización de la Comunidad en Medellín (Martínez, 1981).

Para este mismo año se fundó la Cooperación Social Campesina, entre 1955 y 1956 se creó la Escuela Rural, el Centro Rural de jóvenes y la Residencia Social León XIII; estos fueron algunos de los centros de prácticas para las asistentes sociales para atender las demandas de la población rural (Sandino, 1987).

La implementación de políticas de vivienda en Antioquia respondió al incremento de la población urbana que reflejaban los censos, aunque la población rural aún era mayoría (Melo, 1988). El impulso al sector de la construcción derivó en mayor concentración de capitales y cobro de impuestos, y el aumento de la propiedad privada en la ciudad.

Para contrarrestar la problemática de vivienda se implementó en 1955 "Una política de vivienda en Colombia”, programa que fue llevado a cabo con el Instituto de Crédito Territorial 
(ICT), la banca y el sector privado, donde la participación de los trabajadores sociales estuvo presente en la dinamización de estudios socio-económicos de las familias para la entrega de estas (Martínez, 1981) y "en procesos de rehabilitación de barrios, autoconstrucción y erradicación de tugurios" (Escobar, 1995, p. 135). Además, bajo el concepto de "servicio social industrial”, las trabajadoras sociales eran llamadas a estimular a los obreros a que adquirieran sus viviendas propias por medio de créditos. Por lo anterior, se considera que el TS, desde su génesis, había sido impulsador de políticas de vivienda.

En 1954 se creó el nuevo código procesal del trabajo como reivindicación de las luchas desde la organización sindical obrera, y se organizó el sistema pensional del país (Sandino, 1987). En 1955, durante la dictadura militar de Rojas Pinilla (1953-1957), se reglamentaron las escuelashogar para campesinas, que venían funcionando desde 1947 y se dieron beneficios económicos para la educación superior privada.

En la década del 50, las actividades económicas en Antioquia estuvieron basadas en el impulso de la ganadería y la agricultura por medio de adjudicación de créditos y campañas agrícolas (El Colombiano, 1950c, 1951d). En el marco nacional, se recurrió a actividades especulativas para contribuir "al desarrollo de una agricultura de tipo comercial” (Martínez, 1981, p. 75). Así, la burguesía ve en la explotación de la tierra y la tecnificación de sus procesos, una forma de fortalecer los procesos productivos dentro del concierto mundial.

Para lograr dichos fines, el partido conservador, en alianza con la iglesia católica, utilizó la incautación de tierras a los campesinos por la acción violenta o de manera sutil por medio de préstamos hipotecarios, a través de la Caja de Crédito Agrario Industrial y Minero (Martínez, 1981).

En 1953 la radicalización del movimiento campesino surgió como nueva propuesta de reiniciación desde la organización del campesinado (Martínez, 1981), en 1954 se aprobó el voto femenino y más tarde, en el año 1957, se fundó el Movimiento Revolucionario Liberal (MRL) como oposición al Frente Popular, fundado por Alfonso López Michelsen (Marín y Aguirre, 2013).

También, para esta década empezó a tener acogida la organización comunitaria y, en 1958, bajo el primer gobierno del Frente Nacional, fue promulgada la ley que fomentó la acción comunal, en la que se organizan los campesinos y habitantes de barrios (Sandino, 1987).

Esto acompañado de la aplicación de medidas de corte keynesiano, al crear bancos como el Popular, el Cafetero y el Ganadero, como necesidad de subsidiar la demanda. Todo esto por recomendaciones del Banco Mundial y la banca internacional. Antioquia tuvo injerencia importante en el desarrollo del sector bancario y crediticio, siendo que en 1961 se crea la Bolsa de Valores de Medellín (Melo, 1988). 
Más allá de la caridad y la filantropía: el surgimiento del Trabajo Social en Antioquia

Como parte del desarrollo del TS para 1951, se dio la creación de la Asociación de Escuelas y Facultades de Trabajo Social ${ }^{7}$ (Martínez, 1981), como propuesta que buscaba fortalecer la formación en cuestiones teóricas, metodológicas y epistemológicas. En 1952 se creó la Asociación de Trabajadores Sociales de Antioquia (ATSA), con la participación de 16 egresadas, cinco de ellas de la Escuela anexa al Colegio del Rosario de Cundinamarca, Stella Jaramillo y Blanca Cadavid. Al continuar con el impulso de la profesión, en 1957 se hace el I Seminario de Escuelas de Trabajo Social en agosto, en Bogotá.

En 1953 se conforma la tercera escuela del país en Cali, y mientras tanto, la Escuela en Medellín se articula a la Universidad Pontificia Bolivariana en 1955. En Bogotá, en 1956 se clausura la Escuela de Servicio Social, anexa al Colegio Mayor de Nuestra Señora del Rosario.

En 1960 la Escuela, por aceptación del Gobierno Nacional, pasa a la categoría de "Facultad de Servicio Social" con un pensum de cuatro años que avanza significativamente en la visión general de las Ciencias Sociales y Humanas [con cursos como Antropología Cultural, Problemas Sociales, Filosofía Social y Economía Colombiana] con un marcado enfoque psicologista, acompañado de una formación neutral y objetiva. (UPB, 1995, p. 128)

De este modo, se consideró como punto de referencia para la creación de las demás escuelas nacionales (Montoya, 2005).

Esta década se caracteriza por una formación teórico-práctica y una fortaleza en las metodologías de trabajo de caso, grupo y comunidad, al mismo tiempo que se incluyen algunos conocimientos sobre Política Social y Bienestar Social, al lado de las primeras aproximaciones a la investigación y administración social. (UPB, 1995, p. 128)

A pesar de lo anterior, se evidencia la continuidad de las asignaturas planteadas entre los años 1945-1950, con la particularidad del cambio de materias de tipo doméstico, prosiguiendo la formación moral y religiosa (UPB, 1995). Esto bajo supervisión de algunas universidades católicas de Estados Unidos (Jaramillo, 1996).

A partir del decenio de 1960 se establecieron los programas del Instituto Colombiano de Bienestar Familiar, en los cuales los trabajadores sociales eran contratados para desempeñar acciones de administración, asesoramiento, supervisión, ejecución de programas, y protección y prevención de menores en adopción (UPB, 2000). Posteriormente, se extendieron y establecieron las funciones del servicio social en el Instituto Colombiano de Seguro Social (ICSS) y en las entidades estatales como la Policía Nacional y el municipio de Medellín.

${ }^{7}$ Que en 1965 se convertirá en el Consejo Nacional para la Educación en Trabajo Social (CONETS). 
Del mismo modo, "la acción en las empresas asume perfiles comunitarios (...) a través del liderazgo en acciones de organizaciones y promoción comunitaria” (Escobar, 1995, p. 135). Para el "Trabajador Social se crea la necesidad de incluir en su preparación académica las teorías administrativas del manejo del recurso humano” (Sierra, 2005, p. 131).

En el campo de la práctica, el TS en Antioquia, por su origen en el secretariado social de Fabricato, radica sus procesos de intervención cuando se afirman sus funciones en las industrias manufactureras de la ciudad, con la premisa del servicio social industrial: "Levantar el nivel social, mental y cultural del obrero” (Jaramillo, 1996, p. 27). También incidieron en las fábricas que tenían trabajadores sindicalizados de la Unión de Trabajadores de Antioquia (UTRAN) (Sandino, 1987).

Igualmente, se buscó el fortalecimiento y la posición en otros campos de acción de la asistencia social, basados en lineamientos de tipo mecánico y tecnicista en las instituciones de beneficencia, principalmente en el área de la salud, ubicados en las zonas paupérrimas de Medellín. Se desarrollaban diferentes actividades formativas, que según lo que planteaba Gómez (1951), "lo realmente importante no es que la población aprenda, lo indispensable es que esta no incursione en otros espacios indebidos" (p. 5) ${ }^{8}$; se evidencia por lo anterior que las asistentes sociales reproducían los modelos de dominación de las élites que dieron paso alTS, con el fin de mantener el control social, donde la población no representara una amenaza ante estas.

\section{Conclusiones}

Los fenómenos sociales y los hechos históricos no existen aislados e inconexos, separarlos de su contexto social, político, económico y cultural es privarse de analizar ampliamente el panorama de la realidad; el trabajo social no se da entonces desarticulado de estos, si no que se deriva de la realidad social concreta que se dan en situaciones históricas específicas.

El modelo de producción capitalista y las dinámicas que se dan dentro de este, ha causado que profesiones como el trabajo social estén a su disposición para legitimar las relaciones de poder en la sociedad, intensificando la brecha de desigualdad entre las clases sociales, donde la clase subalterna resulta ser la más perjudicada.

En este sentido, el trabajo social ha contribuido como uno de los mecanismos institucionales movilizados por la burguesía e insertado en el aparato burocrático del Estado, de las empresas y otras entidades privadas en la creación de políticas sociales que legitimen el ejercicio del

\footnotetext{
${ }^{8}$ Amanda Gómez realizó una serie de publicaciones en el periódico El Colombiano, entre los años 1947 y 1962, haciendo seguimiento de los acontecimientos más importantes de la ESS, desde su definición hasta sus funciones detalladas en los principales campos de acción; también publicó la columna de "casos sociales", exponiendo problemáticas comunes y su posible solución desde elTS.
} 
Más allá de la caridad y la filantropía: el surgimiento del Trabajo Social en Antioquia

poder de clase, contraponiéndose a las iniciativas propias de organización y representación de la clase trabajadora.

Por último, se hace evidente la necesidad de trascender la enseñanza de la metodología del trabajo social clásico y sus teorías, superando su realidad histórica en términos de asistencia social, dando paso a otras perspectivas, que propicien la reflexión y el análisis de una profesión con una apuesta ético-político a favor de la clase trabajadora.

\section{Referencias}

Acanda, J. (2004). Educación, Ciencias Sociales y cambio social. En M. Romero. (Ed.), Concepción y metodología de la educación popular. Selección de lecturas. Tomo I (pp. 21-34). La Habana, Cuba: Caminos.

Botero, S. (2006). La reforma constitucional de 1936. El Estado y las políticas sociales en Colombia. Anuario Colombiano de Historia Social y de la Cultura, (33), 85-109.

Brando, C. (2017). Mariano Ospina Pérez, la Caja Agraria y la construcción del Estado. Recuperado de http: / / www.banrep.gov.co/es/sse-bogota-467

Castañeda, M. (2016). Dinámicas territoriales en el municipio de Mutatá, Colombia a partir de la explotación mineral de oro. Recife, Brasil: Universidad Federal de Recife.

Castañeda, M. y Ramírez, D. (2011). Encuentros y rupturas en la historia del Trabajo Social en Brasil y Colombia. Revista Trabajo Social, (13), 9-31.

De la Garza, E. (1993). Postmodernidad y totalidad. Revista Mexicana de Sociología, (4), 131 146.

Los gastos forzosos de los municipios. (30 de julio de 1920). El Colombiano, p.3.

Durante el año 1945 fueron presentados 32 pliegos obreros de peticiones en Antioquia. (8 de enero de 1944a). El Colombiano, p. 2.

Se crean en Medellín nuevos centros pedagógicos para los niños y las niñas. (3 de febrero de 1944b). El Colombiano, p. 9.

Sociedad para explotación de metales acaba de fundar en Medellín \$20.000 de capital. (17 de febrero de 1944c). El Colombiano. p. 3

Repetidas protestas universitarias en Medellín. (23 de marzo de 1944d). El Colombiano, p. 3.

Una sola mina de Antioquia producirá la mitad de oro de Colombia en mayo. (26 de mayo de 1944e). El Colombiano, p. 6. 
La juventud tradicionalista de Antioquia recibirá una nueva organización política. (19 de junio de 1944f). El Colombiano, p. 3.

Solemnemente fue inaugurado el pabellón infantil del hospital. (7 de julio de 1944g). El Colombiano, p. 10.

El balance de Fabricato demuestra los progresos de esa poderosa empresa. (19 de julio de 1944h). El Colombiano, p. 4.

Alza general de salarios piden todos los obreros municipales; por fin se instaló el concejo. (22 de agosto de 1944i). El Colombiano, p. 1.

El directorio conservador de Antioquia ordena combate sin cuartel contra el comunismo .)(30 de agosto de 1944j). El Colombiano, p. 4.

Campaña de protección infantil se iniciará en el departamento.(30 de agosto de 1944k). El Colombiano. p. 5.

Guerra a la invasión comunista piden conservadores de Cisneros. (31 de agosto de 1944l). El Colombiano, p. 9.

Fue reglamentado el pago de las prestaciones sociales a los trabajadores departamentales. (7 de septiembre de 1944m). El Colombiano, p. 2.

Grave agitación social están provocando los comunistas de Antioquia. (11 de septiembre de 1944n). El Colombiano, p. 2.

Los trabajadores del Departamento piden aumento de salarios. (29 de julio de 1945a). El Colombiano, p. 4.

500.000 pesos para construir casas de campesinos destinará la asamblea; listo el proyecto. (29 de agosto de 1945b). El Colombiano, p. 2.

Se fabrica pavimento en Medellín. (31 de agosto de 1945c). El Colombiano, p. 2.

Se activa la campaña pro-casa propia para empleados y obreros. (24 de octubre de 1945d). El Colombiano, p. 2.

Moderno equipo de maquinaria adquirió Tejares Niquia para dotar sus talleres en Bello. (enero de 1946a). El Colombiano, p. 2.

El Colombiano. (13 de febrero de 1946b). El Colombiano, p. 2.

Servicio gratuito médico y dental tendrán los vecinos de algunos corregimientos. (27 de febrero de 1946c). El Colombiano, p. 1.

"Coltejer" montará gran plataforma para producir hilaza de rayón. (26 de abril de 1946d). El Colombiano, p. 13. 
Más allá de la caridad y la filantropía: el surgimiento del Trabajo Social en Antioquia

Se lanzan piedras a Gaitán en el discurso que dio en el Parque Berrio. (10 de septiembre de 1946e). El Colombiano, p. 9.

El Colombiano. (1 de febrero de 1947a). Grandes concentraciones conservadoras en Antioquia. El Colombiano, p. 1.

Plan obrero subversivo denuncia el gobernador "paro imprevisto injusto preparan las entidades sindicales en Antioquia. (3 de febrero de 1947b). El Colombiano, p. 2.

Se inicia protección materno-infantil en las zonas pobres de Medellín. (19 de marzo de 1947c). El Colombiano, p. 7.

Antioquia ha logrado su mayor cifra en la votación conservadora. (9 de abril de 1947d). El Colombiano, p. 1.

Sancionados con la destitución todos los empleados desleales del ferrocarril en Antioquia. (15 de mayo de 1947e). El Colombiano, p. 1.

Más de 50 miembros del sindicato del municipio capturados por la policía. ( 9 de septiembre de 1947f). El Colombiano, p. 2.

La ciudad será consagrada como capital conservadora. (4 de octubre de 1947g). El Colombiano. p. 10.

Acuerdo anticomunista para la preservación de la democracia. (20 de abril de 1948a). El Colombiano, p. 6.

Colombia rompió relaciones con Rusia. Inmediatamente tendrán que abandonar el país los agentes soviéticos. Queda reprimida la acción roja en nuestro país. (4 de mayo de 1948b). El Colombiano, p. 1.

El conservatismo en Antioquia apoya el frente anticomunista. (27 de mayo de 1948c). El Colombiano, p. 1.

Serán canceladas las matrículas de los estudiantes huelguistas. (27 de mayo de 1948d). El Colombiano, p. 1.

El partido liberal niega su pasado histórico y rechaza el frente nacional anticomunista. (29 de mayo de 1948e). El Colombiano, p. 3.

Organizadas las masas derechistas de universidades. (22 de junio de 1948f). El Colombiano, p. 8.

Obligación de consumir las materias primas en Colombia ordena el gobierno a las industrias. (5 de agosto de 1948g). El Colombiano, p. 8.

Ridícula manifestación de los comunistas fracasó ayer en las calles de Medellín. (25 de febrero de 1949a). El Colombiano, p. 2.

Desórdenes estudiantiles anoche. Huelguistas y policías fueron heridos en choques. El ejercito tuvo que salir a imponer el orden. (16 de marzo de 1949b). El Colombiano, p. 1. 
El gobierno seguirá firme frente a la huelga de los universitarios en Antioquia. (29 de marzo de 1949c). El Colombiano, p. 2.

El conservatismo se opone a la propagación de la tesis soviética. (5 de mayo de 1949d). El Colombiano, p. 8.

La escuela católica es un derecho natural que tiene la santa iglesia. (8 de julio de 1949e). El Colombiano, p. 3.

Viajan a España dos estudiantes de Servicio Social de la ciudad de Medellín. (2 de enero de 1950a). El Colombiano, p. 9.

236 jóvenes comunistas detenidos en Medellín. (31 de mayo de 1950b). El Colombiano, p. 12.

Importante reunión ha realizado en Medellín la caja de crédito agrario. (15 de junio de 1950c). El Colombiano, p. 2.

La escuela de servicio social en Medellín (recuento histórico). (16 de julio de 1950d). El Colombiano, p. 3.

Los comunistas y la C.T.C. (6 de octubre de 1950e). El Colombiano, p. 13.

Inscripciones en la escuela de Servicio social. (24 de enero de 1951a). El Colombiano, p. 10.

Escuela de Servicio Social en Medellín, abiertas matrículas en su nuevo local, calle Pichincha. (9 de febrero de 1951b). El Colombiano, p. 1.

Escuela de Servicio Social de Medellín anuncia la iniciación de un nuevo curso, nutrición. (28 de mayo de 1951c). El Colombiano, p. 10.

El crédito agrícola será una de las medidas más eficaces en la nueva política económica. (8 de junio de 1951d). El Colombiano, p. 7.

La escuela de Servicio Social brindará un reconocimiento a la señorita Marta Ezcurra (jefe de la sección de servicio social de la unión panamericana) por sus labores de Servicio Social industrial. (26 de junio de 1951e). El Colombiano, p. 1.

Escobar, H. (1995). Facultad de Trabajo Social, cincuenta años construyendo bienestar. Revista de la Facultad de Trabajo Social UPB, 12(12), 133-136.

Fajardo, N. (2013). Hacia una caracterización de la formación socioeconómica colombiana. Bogotá, Colombia: Documento de estudio.

Gómez, A. (25 de febrero de 1951). El Servicio Social Industrial. El Colombiano, p. 5.

Gómez, C.L. (2001). Breve historia de Antioquia. Medellín, Colombia: Universidad de Antioquia.

Gramsci, A. (2002). Cadernos do cárcere. Rio de Janeiro, Brasil: Civilização Brasileira.

Jaramillo, S. (1996). Antecedentes, fundación y evolución histórica de la Escuela de Servicio Social, anexa 
Más allá de la caridad y la filantropía: el surgimiento del Trabajo Social en Antioquia

a la Normal Antioqueña de Servicio Social. Medellín, Colombia: Universidad de Antioquia.

Lenin, V. (2003). El Estado y la revolución. S.I., Argentina: Unión de Juventudes por el Socialismo.

Marín,A.L.yAguirre,S.(2013). Sentidos y significados del movimiento dela Reconceptualización del Trabajo Social en las unidades académicas de Caldas y Cundinamarca en Colombia. Eleuthera, 9 (2), 211-235.

Martínez, M.L. (1981). Historia del Trabajo Social en Colombia 1900-1975. Bogotá, Colombia: Tecnilibros LTDA.

Marx, K. y Engels, F. (1999). Manifiesto del Partido Comunista. Recuperado de https://www. marxists.org/espanol/m-e/1840s/48-manif.htm

Medina, M. (1984). La protesta urbana en Colombia en el siglo XX. Colombia, Colombia: Ediciones Aurora.

Melo, J.O. (1988). Historia de Antioquia. Medellín, Colombia: Presencia Ltda.

Montoya, A. (2005). Trabajo Social y el Desarrollo Académico en la Facultad de Trabajo Social: 1945-200. Revista de la Facultad de Trabajo Social UPB, 21(21), 97-119.

Quintero, S. (2014). El “Método Caldas”: una expresión profesional de la lucha de clases en el capitalismo latinoamericano (tesis de maestría). Universidad Federal de Río de Janeiro, Río de Janeiro, Brasil.

Ramírez, D. (2014). La utopía de la emancipación humana en Colombia: partidos de izquierda y sindicatos en el Gobierno de Álvaro Uribe Vélez (2002-2010). São Luís do Maranhão, Brasil: Universidade Federal do Maranhão.

Renaud, A. (2015). Espacio y territorio, la necesidad de una definición crítica. Fanzine Hebdomas, (4).

Sandino, M. (1987). Origen y evolución histórica de la práctica de Trabajo Social en Antioquia (Tesis). Universidad de Antioquia, Medellín, Colombia.

Sierra, A. (2005). El trabajo social en las organizaciones: haciendo historia. Revista de la Facultad de Trabajo Social UPB, 21(21), 129-133.

UPB. (1995). Facultad de Trabajo Social 50 años. Revista de la Facultad de Trabajo Social UPB, $12(12), 128-136$.

UPB. (2000). El ejercicio profesional de los egresados de la Facultad de Trabajo Social de la UPB. Revista de Trabajo Social UPB, 17(17), 10-24.

Vega, R. (2002). Gente muy rebelde. Bogotá, Colombia: Pensamiento crítico. 